\title{
Eliciting Tacit Expertise in 3D Volume Segmentation
}

\author{
Ruth West \\ Meghan Kajihara \\ Max Parola \\ Univ. of North Texas \\ Michelle Holloway \\ Wash. Univ. in St. Louis
}

\author{
Kathryn Hays \\ Luke Hillard \\ Anne Carlew \\ Univ. of North Texas \\ Brendan John \\ Rochester Inst. of Tech.
}

\author{
Jeremey Deutsch \\ Brandon Lane \\ Univ. of North Texas
}

Anahita Sanandaji
Cindy Grimm
Oregon State University

\begin{abstract}
The output of $3 \mathrm{D}$ volume segmentation is crucial to a wide range of endeavors. Producing accurate segmentations often proves to be both inefficient and challenging, in part due to lack of imaging data quality (contrast and resolution), and because of ambiguity in the data that can only be resolved with higher-level knowledge of the structure and the context wherein it resides. Automatic and semi-automatic approaches are improving, but in many cases still fail or require substantial manual clean-up or intervention. Expert manual segmentation and review is therefore still the gold standard for many applications. Unfortunately, existing tools (both custom-made and commercial) are often designed based on the underlying algorithm, not the best method for expressing higher-level intention. Our goal is to analyze manual (or semi-automatic) segmentation to gain a better understanding of both low-level (perceptual tasks and actions) and high-level decision making. This can be used to produce segmentation tools that are more accurate, efficient, and easier to use. Questioning or observation alone is insufficient to capture this information, so we utilize a hybrid capture protocol that blends observation, surveys, and eye tracking. We then developed, and validated, data coding schemes capable of discerning low-level actions and overall task structures.
\end{abstract}

\section{Keywords}

$3 \mathrm{D}$ volume segmentation; conceptual framework

\section{INTRODUCTION}

$3 \mathrm{D}$ volume segmentation is an important and ubiquitous task in image-oriented scientific disciplines, such as biology, environmental science, or medicine, where volume images provide the basis for discovery. While imaging reveals spatial content and activities within an entire subject, ultimately researchers and clinicians are interested in specific

Permission to make digital or hard copies of all or part of this work for personal or classroom use is granted without fee provided that copies are not made or distributed for profit or commercial advantage and that copies bear this notice and the full citation on the first page. Copyrights for components of this work owned by others than the author(s) must be honored. Abstracting with credit is permitted. To copy otherwise, or republish, to post on servers or to redistribute to lists, requires prior specific permission and/or a fee. Request permissions from permissions@ acm.org.

VINCI '16, September 24 - 26, 2016, Dallas, TX, USA

(C) 2016 Copyright held by the owner/author(s). Publication rights licensed to ACM. ISBN 978-1-4503-4149-3/16/09. . \$15.00

DOI: http://dx.doi.org/10.1145/2968220.2968235
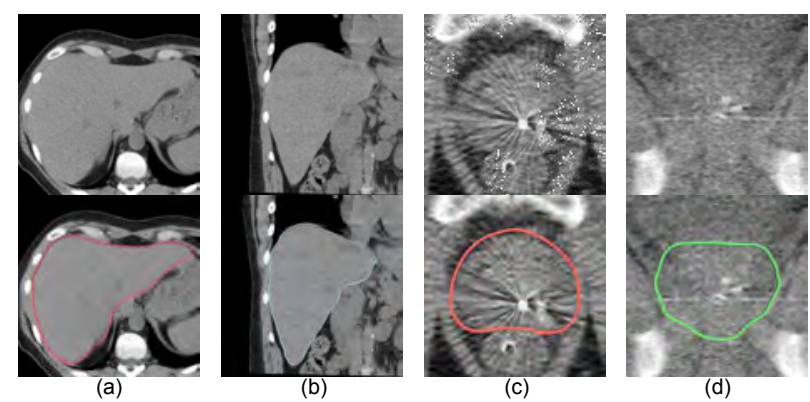

Figure 1: Example image slices of 3D volumetric data with contours marked. a) and b) are CT liver scans, c) and d) are prostates.

structures within volumes (e.g., organs, tissues, cells, $\mathrm{CO}_{2}$ filled fractures within materials etc.) for purposes such as visualization, quantitative analysis, or physical computation and simulation. The delineation of 3D structures of interest within volume imaging data is therefore a typical first-step in the data-to-knowledge pipeline, and both the efficiency and accuracy of segmentation critically affect how the data is utilized in research and clinical practice.

Creating accurate segmentations, particularly for 3D biomedical images, is a highly non-trivial task that calls for cooperation between humans and computers. Segmentation involves making many local decisions based on image features (e.g. boundaries of biological tissues are not always clearly separated), potentially spatially and topologically complex 3D structures and resolving issues due to low image resolution or noise (see examples in Figure 1). Segmentation times can range from a few minutes to days to weeks.

While human experts, with their superior visual perception skills and domain knowledge and experience acquired from years of training, ultimately decide what constitutes an accurate segmentation, they lack the objectivity or efficiency of computational algorithms. Additionally, segmentation is usually performed (or evaluated) on 2D slices of the 3D data and the segmenter must mentally integrate these into a coherent 3D structure. Methods for automatic segmentation are becoming more reliable [26] yet many segmentations are still created or reviewed manually to ensure the necessary quality and accuracy, as without expert guidance segmentation algorithms easily fail in the presence of noise and ambiguity within the data. Thus experts create $3 \mathrm{D}$ segmentations using low-level perceptual cues to perform low-level 
marking, all guided by higher-level cognitive tasks and domain knowledge. Low-level cues include texture or spatial attributes [23], low-level marking tasks include delineating structures in an image plane by marking contours or filling regions. Experts also use higher-level constraints - such as connectivity, shape and topology - to disambiguate unclear structure boundaries [13].

Despite the large amount of work on developing interactive and semi-automated segmentation tools, there is little research on the correct way to design and evaluate such systems, nor is there research on how to capture, in a finegrained way, what experts are doing in the $3 \mathrm{D}$ segmentation process. Our work looks at $3 \mathrm{D}$ volume segmentation as a human-computer interaction paradigm with the ultimate aim of understanding the human factors involved in current segmentation processes. Our goal is to make the process more efficient, accurate and repeatable. Here we report on progress towards developing methods to better understand how humans perform low-level perception and cognition tasks in the context of 3D segmentation (e.g., visual cues, delineation of structures by marks, and local accuracy or quality criteria) and how domain experts wish to specify high-level segmentation constraints (e.g., connectivity, topology, and shape).

Tools and interfaces used in 3D segmentation are often designed around underlying algorithms and input devices. Their complexity requires segmenters to develop significant expertise in a given tool, its underlying rationale, and the constraints it imposes on workflow irrespective of the data or structure being segmented. Thus expertise is situated within a combination of tool use, workflows, and domainspecific knowledge. Our goal is to elucidate commonalities in workflow and expertise skill sets across use contexts, essentially extracting tacit knowledge from within idiosyncratic workflows operating in domain-specific environments. Unfortunately, removing segmenters from their workflow context (e.g., providing a generic segmentation task) essentially strips experts of their expertise and transforms all study participants into novices. While design of generic tasks utilizing synthetic data sets is a useful strategy for future controlled studies, the data, tasks and downstream uses of the resulting geometries from 3D segmentation in the field are sufficiently complex that constructing a synthetic data set is not only difficult, but may not yield sufficient insight at this formative stage of our research. In brief, our research addresses the need for methods to understand - on a fine grained level - what experts are doing during $3 \mathrm{D}$ volume segmentation, to elucidate lower level cognitive and perceptual tasks, higherlevel constraints and behaviors and mental models in order to improve the accuracy, repeatability and quality of segmentation. Our objective is to develop approaches applicable to the process of $3 \mathrm{D}$ segmentation irrespective of the domain (or tool set) in which it is applied.

Our hypotheses are: 1) elucidating low level segmentation processes (e.g., visual cues, mark making and placement, local criteria for accuracy or quality etc.) can inform the development and quantitative evaluation of methods for improving the segmentation process irrespective of application domain; and 2) elucidating higher-level segmentation processes and constraints can establish a set of desirable higher-level behaviors, eventually leading to development of design heuristics for segmentation processes, training paradigms, tools, and algorithms to produce those be- haviors.

Our contributions include: 1) design of a hybrid protocol (see figure 2) for formative studies for extracting what is essentially tacit expert knowledge within the application domain of $3 \mathrm{D}$ volume segmentation, and 2) applying this protocol to identify different segmentation approaches utilized by expert versus novice segmenters in the field.

\section{RELATED WORK}

Ample evidence elucidates the ways in which current segmentation and visualization process are both inefficient [12], and lack repeatability $[7,18,2,10,25,24]$, and the ways in which resulting geometries (surfaces) often require a substantial amount of editing (e.g., fixing topology and connectivity) before being suitable for their intended use. Substantial work has focused on creating and evaluating various 3D image segmentation methods but only a few have taken into account humans and their role. As in recent work by Ramkumar et al. [21] investigating the effects of user interaction in semi-automatic segmentation methods for segmenting organs at risk in radiotherapy planning, the focus of human-computer interaction research and design of segmentation tools and processes reflect the mode of data capture, the logic of underlying algorithms (with each new algorithm adding functionality), and input devices with the aim of providing flexibility in user interfaces or decreasing the cognitive load of interactions within overall process. The focus of HCI optimization ultimately arises from the concept of "fewer clicks", translating to ease of use rather than from the notion of domain knowledge as supportive of ease of use. Olabarriaga and Smeulders [20] presents an early review of human-computer interaction in volume segmentation, providing three common criteria for segmentation evaluation: accuracy, repeatability and efficiency. Krupinski [15] seeks to understand how physicians perceptually interact with the information in medical images during the interpretation process, with an emphasis on visual search for anomaly, or region-of-interest, detection. While these are applicable to 3D segmentation, they represent a subset of what is required to reason and act upon $3 \mathrm{D}$ volume data in order to extract $3 \mathrm{D}$ structures. In order to assess the quality of differences in expertise in the comprehension of medical visualizations Gegenfurtner et al. [8] reviews quantitative studies that characterize where experts look based on where in the image their gaze rests (fixation) and how long it is there (dwell time). Fixation and dwell time are lower level tasks that relate to higher order tasks of searching, abnormality detection, and accuracy of detection. Segmentation, while requiring the ability to detect a feature of interest, also requires the expert to build a 3D structure from those features. Recent work by Li et al. [17] lays the groundwork for assessing differences in experts' comprehension of visualization, but not for 3D segmentation.

Prior knowledge and experience are important elements for the problem solving inherent in 3D segmentation. The cognitive structures (mental models or schemata) thought to underlie $2 \mathrm{D} / 3 \mathrm{D}$ imaging data interpretation unify a segmenter's competence (in the Chomskyan sense) in that they link knowledge of their domain and segmentation praxis with their actual segmentation behaviors in situ. These structures allow segmenters to draw on previous experiences and knowledge to guide their visual problem solving, reasoning, and actions upon $3 \mathrm{D}$ volume data, which is often pre- 


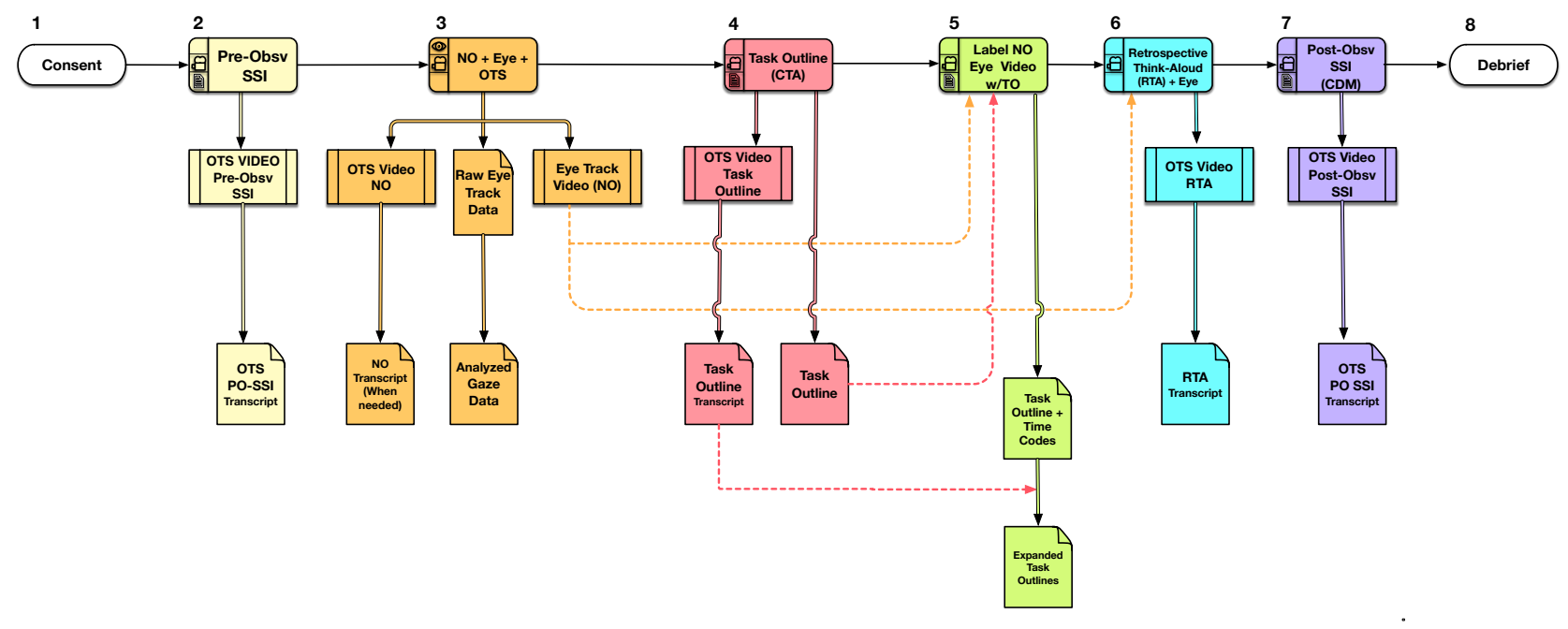

Figure 2: Protocol phases and data products: CTA, CDM, RTA and eye movement tracking are combined with semi-structured interview to yield task outlines, eye gaze analysis, and coded transcripts.

sented to them as a series of $2 \mathrm{D}$ slices through a volume. Neisser [19] proposed the concept of an "adaptable" schema which drives the visual exploration of a scene for imaging interpretation. Visual information feeds into and modifies schemata which further direct visual exploration of a scene. This process is cyclical, and the process of schema access and/or development, from retinal detection to cortical perception, in visual search and detection is quite rapid. Thus each segmenter can be thought of as imposing unique biases on the image based on prior knowledge, experience, and expectations, which can be helpful in decoding the imaging data (composed of colored 3D pixels (voxels)) and making decisions about visual objects. Though not sufficient to understand the full complement of processes at play during 3D segmentation, schemata/mental models are necessary for understanding the complex interplay of perception, cognition and domain knowledge in 3D segmentation workflows.

\section{HYBRID PROTOCOL DESIGN}

We first describe the motivation behind the protocol, and the elements we combine and why (Section 3.1). We next describe the actual field capture procedure and the data collected (Section 3.2, the participants 3.3, the semi-structured interviews (Section 3.4), and guiding annotation codes used to structure the interviews (Section 3.4).

\subsection{Protocol design elements and motivation}

Through informal interaction with segmentation experts and novices we developed an initial understanding of the kinds of limitations and problems encountered in the field. Existing segmentation practices, whether accomplished via commercial software packages or custom tools, share a common iterative structure that incorporates inspection, navigation, marking, editing, and visualization. Each of these overarching tasks requires multiple sets of sub-tasks, each with their unique sets of constraints and challenges. Our protocol builds upon our knowledge of the problem domain and its design seeks to elicit tacit expert knowledge through observations in the field while segmenters are utilizing a repre- sentative complement of tools and data that they encounter during routine research or clinical practice. The aim is to not only understand segmentation experts, but also how novices differ from them.

A distinguishing feature of $3 \mathrm{D}$ volume segmentation is that the characteristics of the data vary according to the imaging modality by which it was produced, and often have no resemblance to how the imaged subject might appear in the real world. As segmenters work their way through the data they are constantly evaluating new visual stimuli based on what they just annotated. While many other complex workflows may use visual representations as an aid (e.g., train scheduling or network intrusion detection [6]) segmentation can not be accomplished independent of visual reasoning.

To address these characteristics of segmentation, and the fact that expert knowledge becomes automatized and therefore more difficult to elicit, we developed a hybrid protocol that blends elements from existing qualitative and quantitative human-computer interaction methods so that it can be utilized in the field for formative studies and later combined with additional studies under more controlled conditions. Given that cognition and perception are linked [8], in studies where eye motion tracking is required, the use of a Think Aloud protocol while capturing eye movement as a participant is performing segmentation is ineffective as it affects the participant's eye motion and locus of attention during task performance. We therefore devised a protocol that combines elements of Retrospective Think Aloud (RTA) [5, 11], Cognitive Task Analysis (CTA) [3], Critical Decision Method (CDM) [14], and Eye Movement Tracking (ET), with ethnographic semi-structured qualitative interviews (SSI).

We briefly describe each of these elements, then how we use combinations of them to elicit information at both the low-level and the high. Facilitator roles are summarized in Table 1, and the overall protocol flow in figure 2. RTA is a variant of the "think aloud" protocol that has users provide a task analysis (TA) after a task session rather than during it in hopes of having a more accurate task session. We utilize eye gaze overlay to stimulate the RTA $[11,9]$. 


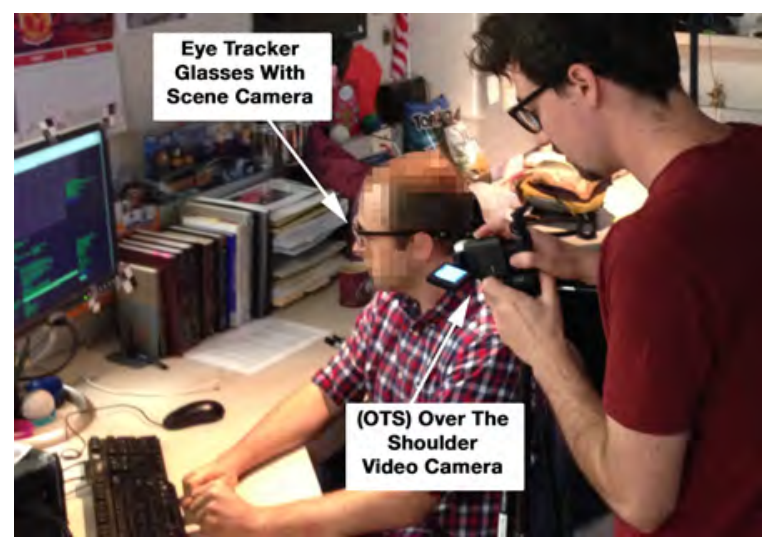

Figure 3: Over the shoulder video captures a view of participant display, keyboard and mouse actions that augments the eye tracker scene camera view.

CTA goes beyond standard task analysis methods to externalize knowledge, thought processes, and objectives that underlie observable behavior (tasks performed). The CTA protocol is based on "cognitive task analysis" using the "concepts, processes, and principles" model [3]. We work with participants to tie the CTA to the observed eye-gaze overlay. CDM is a semi-structured ethnographic method that reduces reliance on individual memory by guiding the participant through a series of multiple passes of event retrospection [14]. CDM seeks to identify cues and other information that enables experts to identify critical events and informs their decisions at these points. We use CDM to elicit higherlevel decision making not apparent in the CTA.

ET: The posited link between eye movement and thought [8] provides a method for revealing what experts are looking at during the process of $3 \mathrm{D}$ volume segmentation.

SSI: Semi-structured interviews utilize a set of topics/themes to guide the conversation across relevant topics without interrupting the flow [1]. We use SSIs in the pre and post interview stages in order to ensure that we cover all topics identified as relevant to segmentation (see Section 3.4).

We use combinations of these elements in the following way: RTA and CTA are blended in order to reveal conceptual and procedural knowledge, cognitive processes and notions of quality or standards used to evaluate segmentation outcomes. We consider low-level actions to be something we can see or hear in the video/audio/ET data rather than actions we think or assume the participant is doing. We use replay of the the eye movement video and CDM to elicit from participants links between lower level (visuals and marks) and higher level (perceptual and cognitive) tasks and behaviors (also known as RTE [11, 5], and found in [9]). This blend of direct observation, eye motion tracking combined with RTA, a prompted multi-pass recall structure similar in format to CDM, and semi-structured interviews enables us to create linkages between thought processes and observable behaviors. We next describe the hybrid protocol as performed on-site.

\subsection{Field-study data capture}

The field study protocol is designed for observing participants (experts and novices) in their work settings with participants using their own tools, data, and workstations. We

\begin{tabular}{|ll|}
\hline Facilitator Roles & Tasks \\
\hline Primary Interviewer & $\begin{array}{l}\text { Conducts the interview/ observa- } \\
\text { tion } \\
\text { Secondary Interviewer } \\
\text { Tracks topic coverage, asks } \\
\text { follow-on questions } \\
\text { Captures video, audio and eye } \\
\text { tracking }\end{array}$ \\
\hline
\end{tabular}

Table 1: Facilitator Roles and Tasks

perform the capture in situ in order to capture the heterogeneity in participant's idiosyncratic workflows. Duration of sessions is approximately 1 to 2 hours maximum. The observation set up (see figure 3) includes over-the-shoulder video, audio recording, hand-written participant information, and eye tracking (utilizing an SMI remote $500 \mathrm{~Hz}$ glasses based tracker).

The protocol is facilitated by a team of three on-site researchers: a primary interviewer, a secondary interviewer, and a note taker (see Table 1 ).

The protocol is diagrammed in figure 2 and progresses through 8 phases. Phase $\mathbf{1}$ is informed consent, where the goal(s) of the study are communicated to the participants. From left to right:

Phase 2 [yellow]: is a pre-observation, semi-structured interview (SSI) where the participant describes their segmentation process, data, tools and provides an overview of their workplace setup.

Phase 3 [orange] is the Naturalistic Observation (NO) where the participant performs as much of their complete segmentation process as possible, as naturally as possible while skipping repetitive actions after a few examples, and without talking (eye tracking and over the shoulder video). Calibration of the eye tracker occurs prior to the NO. We monitor the eye tracking during data capture and re-calibrate as necessary upon detection of drift using times when gaze should match cursor location as a guide.

Phase 4 [red] is the CTA where participants hand-write a task outline (TO) on paper that describes the steps of their segmentation process.

Phase 5 [green] combines CTA and CDM. Participants label their NO eye tracker scene video according to the task outline completed in the prior phase. If necessary, they alter their hand-written CTA or capture more NO video if the two do not align.

Phase 6 [blue] is the RTA. Participants are asked to describe what they are thinking as they view (playback) or scrub through the NO video with eye-gaze overlay.

Phase 7 [purple] is the post-observation, CDM that goes into depth on the participant's decision making process using semi-structured interviews. The protocol concludes with a participant debriefing (Phase 8).

Over the Shoulder Video (OTS) with audio is captured throughout the protocol, except phase 1. Eye motion tracking data is captured in phase 3 , and as needed in phases 5 and 6 . During phases 5 and 6 we record the playback of the NO video in order to synchronize the OTS video and audio with where they were looking in the NO video. Data products for all phases are summarized in Table 2.

\subsection{Participants}

We observed 13 participants from 5 different sites (Table 3 ). The number of participants per site ranged from 2 


\begin{tabular}{|c|c|c|}
\hline Phase & Data Type & Use/Analysis \\
\hline $\begin{array}{l}\text { Pre-Obsv } \\
\text { SSI: } 2\end{array}$ & Over the Shoulder Video & $\begin{array}{l}\text { Declarative knowledge of participants segmentation process, data charac- } \\
\text { teristics, tools and downstream uses for segmentation geometries }\end{array}$ \\
\hline NO: 3,5 & Eye Tracking & Identify eye gaze fixation and dwell time during segmentation \\
\hline NO: 3,5 & Eye Tracker Scene Video & Context of eye gaze \\
\hline $\begin{array}{l}\text { ALL }(2,3,4, \\
5,6,7)\end{array}$ & Over the Shoulder Video & $\begin{array}{l}\text { Wide-view context and audio of workspace and behavior during segmenta- } \\
\text { tion process including one or more screens, and keyboard and mouse actions }\end{array}$ \\
\hline $\begin{array}{ll}\text { TO: } & 4 \\
\text { (CTA) }\end{array}$ & Task Outline & Declarative knowledge of participantâĂŹs segmentation process/ workflow \\
\hline $\begin{array}{ll}\text { TOL: } & 5 \\
(\mathrm{CTA} & / \\
\text { CDM }) & \end{array}$ & Task Label & $\begin{array}{l}\text { Video/audio recording marked with beginning and end of discrete task steps } \\
\text { as per Task Outline }\end{array}$ \\
\hline $\begin{array}{l}\text { RTA + Eye: } \\
6\end{array}$ & $\begin{array}{l}\text { Retrospective Think- } \\
\text { Aloud prompted by Eye } \\
\text { Gaze overlay }\end{array}$ & $\begin{array}{l}\text { Semi-structured recall of segmentation process prompted by video/audio } \\
\text { recording and eye gaze overlay }\end{array}$ \\
\hline $\begin{array}{l}\text { Post-Obsv } \\
\text { SSI: } \quad 7 \\
(\text { CDM }\end{array}$ & CDM Interview & $\begin{array}{l}\text { Semi-Structured interview for participant domain and segmentation process } \\
\text { knowledge, decision points and cues (e.g., image quality, downstream tasks, } \\
\text { etc.) - Decision Points, Cues and Domain Knowledge }\end{array}$ \\
\hline
\end{tabular}

Table 2: Data Type and Use/Analysis

\begin{tabular}{|llllllllllllll}
\hline Participant & P1 & P2 & P3 & P4 & P5 & P6 & P7 & P8 & P9 & P10 & P11 & P12 & P13 \\
Site & 1 & 1 & 2 & 2 & 2 & 2 & 3 & 3 & 3 & 4 & 4 & 5 & 5 \\
Domain & Cell & Cell & Cell & Cell & Cell & Cell & Cell & Cell & Cell & Soil & Cell & Rad & Biomed \\
Exp. & $>3 y$ & $>18$ y & $>1$ yr & $>5 y$ & $>3 y$ & $>40 y$ & $<1$ yr & $<1$ yr & $>5$ yrs & $>1$ yr & $<1$ yr & $>5$ yrs & $>3$ yrs \\
Prior Exp. & Yes & Yes & Yes & Yes & Yes & Yes & Yes & Yes & Yes & Yes & Yes & No & Yes \\
Tool & 1 & 1 & 2 & 2 & 2 & 2 & 3 & 3 & 3 & 4 & 3 & 5 & 5 \\
\hline
\end{tabular}

Table 3: Participants, sites, domains (Cell biology, Soil analysis, Radiology, and Biomedical segmenter), experience, prior experience with data set, and which tool/software.

to 4. Tools used ranged from custom-built to commercially available software. Participants were recruited through personal contacts and were selected to cover a range of tasks, data sets, tools, and expertise.

\subsection{Interview questions and annotation codes}

The Naturalistic Observation (NO) eye tracking data and scene camera video along with NO over the shoulder video serve as the foundation of the protocol. This enables us to avoid observational bias as much as possible. However, CDM requires a list of questions to elicit the hows and the whys of participants' decision making. In order to address the needs of CDM, and to ensure that we were as uniform as possible in our data capture across all participants, we developed an initial set of codes and corresponding prompts encompassing segmentation processes end-to-end. These codes were developed from our experience in the field and refined after each observation.

We refer to this overarching code scheme as our "Master Annotation Codes" (MAC). The top tier is subdivided into 16 codes that span from 3D data capture, through segmentation, to downstream uses of segmentation products. These codes include: Image Data Acquisition, Data PreProcessing, Process Strategies, Data Exploration, Marking Stage, Automated Stage, Review/Refinement Stage, Data Post-Processing, Downstream Use, Overall Process, Data Relation to Use Case, Quality Evaluation in Use Context, Navigation Strategies, Surface Understanding, Tools and Quality Control. The code schema becomes more fine grained with each subsequent tier (see Table 4).

\begin{tabular}{|l|l|l|}
\hline MAC Level 1 & MAC Level 2 & MAC Level 3 \\
\hline Marking Stage & Mark Decision & $\begin{array}{l}\text { Decision Image } \\
\text { Feature }\end{array}$ \\
\hline & & $\begin{array}{l}\text { Decision Domain } \\
\text { Knowledge }\end{array}$ \\
\hline & & $\begin{array}{l}\text { Decision Mark } \\
\text { Confidence }\end{array}$ \\
\hline
\end{tabular}

Table 4: Annotation code hierarchy (an example).

The MAC is used to create a series of prompts for all levels of the hierarchy. These prompts are used in the CDM (phase 7 ) and pre-observation (phase 1). The codes are also used during annotation and analysis and to link hypothesis to specific observations. We demonstrate the ability of this coding scheme to illuminate lower-level perceptual cues, low-level actions (e.g. drawing) and high-level (e.g. marking versus reviewing actions) strategies in our recent publication [22].

\section{ANALYSIS}

Data analysis includes quantitative analysis of gaze fixation and dwell time and characterization of eye motion, along with qualitative analysis of video/audio recordings. Eye movement data is analyzed both with the eye tracker manufacturer analysis software (heat map and gaze location overlays) and custom software. We use MaxQDA [16] for qualitative data analysis. Utilizing the MAC coding scheme we perform thematic analysis over transcripts and video to identify themes, decision cues and decision points, and to 
identify behaviors and sub-behaviors (tasks/sub-tasks).

Analysis is accomplished by multiple investigators independently coding the data to achieve intercoder agreement. While we create transcripts of all video and audio recording data we do not perform linguistic or semantic analysis of transcripts. The aim of our analysis is to elucidate relationships between thought, perception and behavior in the context of accomplishing segmentation, it is not to elucidate the structure of thought during participants' verbalization about the process.

Our focus is not on statistical sample analysis but rather on a repeatable coding scheme to identify particular patterns both within and between participants. To determine expert gaze location and low level actions we analyze heatmap/gaze overlays from the NO videos and eye-tracker raw data files. The combined eye tracking and qualitative analysis revealed that low-level actions naturally grouped into five higherlevel action types as: 1) Eye gaze location: participant's eye gaze location (e.g., gaze location on tool vs. data; or boundary vs. region); 2) Global navigation: navigating between slices (e.g., paging or rotating); 3) Local navigation: zooming and panning; 4) Marking: drawing, filling or editing a contour; 5) Review: reviewing a segmentation process or mark. Three of our researchers individually coded the data using MAXQDA. Coding agreement was achieved by viewing all three reviewers' codes on a single video. Each action has a dedicated code. In a recent publication [22] we demonstrated that the protocol and coding scheme effectively captures, in a quantitative manner, the low-level tasks and behaviors and higher-level actions and constraints observed qualitatively during $3 \mathrm{D}$ volume segmentation by experts and novices. To obtain the initial results presented in Section 5 below we analyzed the data using the following methods:

Action frequency analysis: We compute code frequency and average duration of each code interval over the entire observation. Action frequency analysis helps to understand what low-level actions participants take most often and how interleaved these actions are.

Task Outline snapshot analysis: Using selected video segments corresponding to each of the tasks outlined by participants, task outline snapshot analysis identifies what participants are doing during different stages of the segmentation process.

Higher-Level Task-Flow Analysis: Using a combination of participants' handwritten Task Outline, RTA and CDM, higher-level task flow analysis identifies domain-agnostic phases and patterns of actions during segmentation.

\section{INITIAL RESULTS AND HYPOTHESES}

The protocol and data analysis methods have yielded initial results in two areas: 1) where do experts look, what are their low-level actions, and how do gaze patterns change according to tasks during segmentation [22], and 2) what domain-agnostic high-level task patterns are exhibited by expert and novice segmenters. Together these represent progress towards elucidating low and high-level processes and constraints to address the hypotheses in Section 1.

\subsection{Perception and Low Level Actions}

Using the analysis of expert gaze and low-level actions described in Section 4 (above) we verified the data coding scheme can distinguish different patterns of low-level percep- tual actions, gaze patterns, and tasks. Detailed data analysis is presented in [22]. Key insights from these results include: Strategies: Depending on the tool and the data set, participants utilized one of two segmentation strategies: Regionbased (seeing data as regions to be filled or captured) or boundary-based (seeing data as boundaries to be demarcated by drawing contours). Additionally, if participants captured a structure by drawing, they looked more at the boundaries, while filling or placing marks in structures resulted in more gazing at regions.

CTA and ET: Different participants had different task outlines and gaze patterns even if they worked with similar tools and data sets.

Gaze patterns were different in individual/different tasks, but repeated/similar tasks had similar patterns.

\subsection{Domain-agnostic high-level task patterns}

Using the analysis of expert and novice task outlines, task labeling, RTA and CDM described in Section 3 we identified an initial set of domain-agnostic patterns in overall task flows. These point to potential characteristics of expert versus novice task flows. Observed lower-level tasks naturally associate into six higher-level task groups: Setup, Detect/Identify, Tool Usage, Review, 2D Navigation and 3D Navigation. Steps can be single or multi-purpose, and onetime or repetitive. Setup includes tasks such as configuring software tools, data inspection, or setting parameters, Detect/Identify includes locating regions of interest or segmentation targets; Tool Usage includes actions such as marking, editing, or interacting with tool interface elements such as sliders; Review includes error checking, 2D navigation and $2 \mathrm{D}$ views includes navigation within a plane or plane to plane; 3D navigation and 3D views includes navigation utilizing $3 \mathrm{D}$ visualizations or $3 \mathrm{D}$ views.

We visualize tasks and sub-tasks for Participants P1 P9 in figure 4 as colored rectangles within a grid. For each participant, the main task flow is placed on the first row and labeled as "TASK" and sub-tasks are placed on subsequent rows and labeled as sub-task levels 1, 2, or 3 (ST-L1, ST-L2, ST-3). A maximum of three sub-task levels was observed in this set of participants. Each participant's task flow starts at the left-most colored rectangle and proceeds to the right. When a task is branched to a sub-task, the colored rectangle is placed on a subsequent row/grid level. When a sub-task ends, and a new task begins, the new task is placed on the main task flow. Multi-purpose tasks are indicated by a dualcolor rectangle.

Differences in task flows for all Participants (figure 4 and detail in figure 5), for $\mathrm{P} 1$ and $\mathrm{P} 2$, two experts from within the same study site, demonstrate variations that can arise due to differences in spatial and topological attributes of the structures being segmented from within the volume. The pattern for P1 represents a semi-automated segmentation method in which Tool Usage is based on seed placement to drive region growing algorithms, whereas P3 demonstrates a pattern based on drawing contours on a slice-by-slice basis to contour structures in complex spatial and topological relationships not amenable to semi-automated approaches.

\section{DISCUSSION AND FUTURE WORK}

We hypothesize that the majority of the experts' skill sets (not related to tool familiarity) can be captured with the following three sub-skills: 


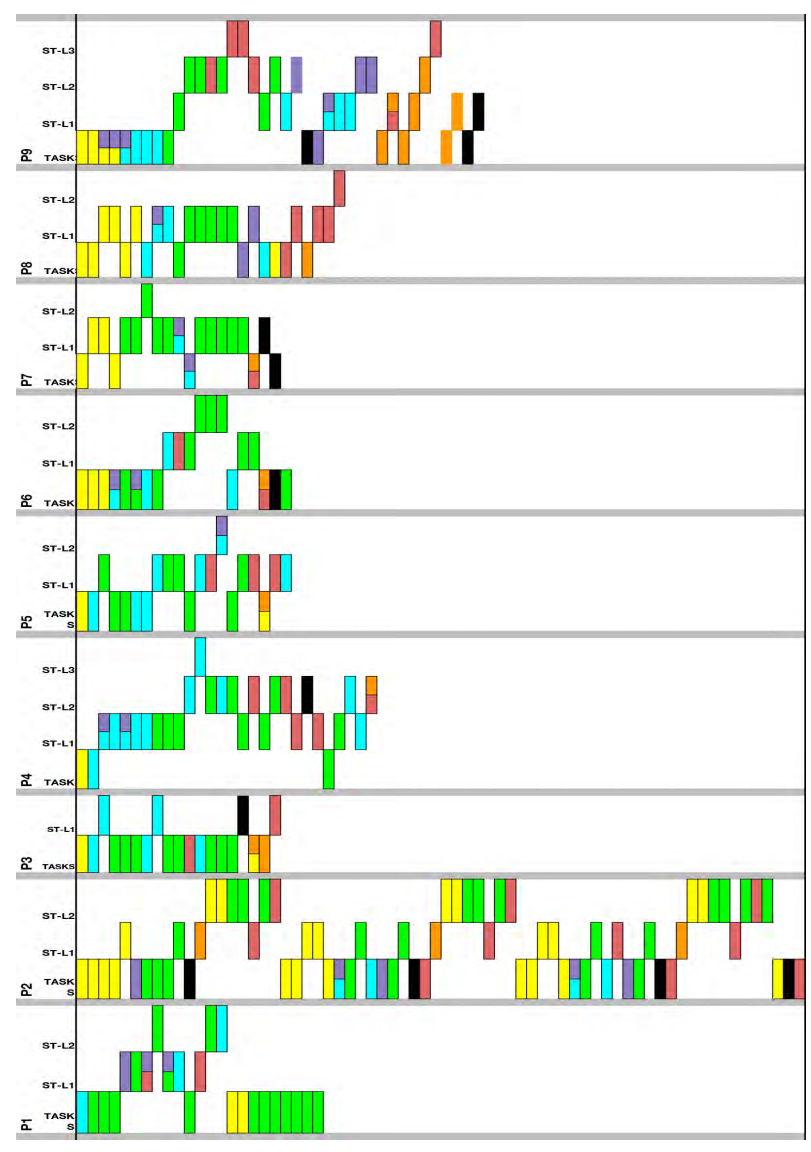

Figure 4: Visualization of domain-agnostic highlevel task patterns Color legend: Yellow (Setup); Blue (Detect/Identify), Green (Tool Usage); Red (Review); Purple (2D navigation and 2D views); Orange (3D navigation and $3 \mathrm{D}$ views). Multi-color blocks are multi-purpose steps. Black are steps that are repetitive.

- Understanding of the 3D shape and its expected variation (surface variation, global shape deformation, branching factor, etc.)

- The ability to predict the expected shapes of the $2 \mathrm{D}$ contours of the 3D shape using (potentially) an arbitrarily oriented slicing direction.

- Visual mapping of the 2D image features (textures, gradients, shading) to expected contour boundaries, i.e., what $2 \mathrm{D}$ image qualities are expected inside, outside, and along the boundary of the contour.

To test this hypothesis we are currently developing a domainagnostic measurement instrument (similar to [4] for items one and two) for these three items. By domain-agnostic we mean the measurement is valid across different $3 \mathrm{D}$ shapes and image qualities; we are simultaneously developing example semi-abstract data sets to capture a wide range of potential structures and image characteristics. Using this instrument and data sets we can evaluate tool-agnostic training methodologies, hopefully separating learning the tool set from learning how to segment.

\section{CONCLUSIONS}

In conclusion, we have presented a hybrid protocol for capturing workflow in the field for a task (3D segmentation) that is highly dependent on both low-level visual processing cues and higher-level domain knowledge. Because the individual expert's skill is linked to both their specific tool and data sets our protocol was designed to capture their workflow in situ. Our subsequent analysis has focused on extracting commonalities that can be observed across these varying domains and tool sets, with the aim of separating tool and data-specific information from fundamental workflow. We have successfully identified both macro-work flow patterns (initial analysis, marking, evaluation) and microsub-task cycles [22] using eye tracking. Analysis of these workflows and mental models has led to a testable domain and tool agnostic hypothesis.

\section{ACKNOWLEDGMENTS}

This research is funded by NSF Grants IIS 1302142, IIS 1302248, DEB 1053554 and DEB 1345795. We thank our participants for all their time and effort, Dr. Reynold Bailey for the use of his eye tracking glasses and his student, and both TOBII and SMI for their loan of their glasses.

\section{REFERENCES}

[1] A. Blandford, D. Furniss, and S. Makri. Qualitative HCI research: Going behind the scenes. Synthesis Lectures on Human-Centered Informatics, 9(1):1-115, 2016.

[2] L. F. Cazzaniga, M. A. Marinoni, A. Bossi, E. Bianchi, E. Cagna, D. Cosentino, L. Scandolaro, M. Valli, and M. Frigerio. Interphysician variability in defining the planning target volume in the irradiation of prostate and seminal vesicles. Radiotherapy and Oncology, 47(3):293-296, 1998.

[3] R. E. Clark, D. Feldon, J. J. van MerriÃńnboer, K. Yates, and S. Early. Cognitive task analysis. Handbook of Research on Educational Communications and Technology, 3:577-593, 2008.

[4] C. A. Cohen and M. Hegarty. Sources of difficulty in imagining cross sections of 3D objects. In Proceedings of the Twenty-Ninth Annual Conference of the Cognitive Science Society, pages 179-184. Cognitive Science Society Austin TX, 2007.

[5] S. Elling, L. Lentz, and M. de Jong. Retrospective think-aloud method: Using eye movements as an extra cue for participants' verbalizations. In Proceedings of the SIGCHI Conference on Human Factors in Computing Systems, pages 1161-1170. ACM, 2011.

[6] T. Farrington-Darby, J. R. Wilson, B. Norris, and T. Clarke. A naturalistic study of railway controllers. Ergonomics, 49(12-13):1370-1394, 2006.

[7] F. Foppiano, C. Fiorino, G. Frezza, C. Greco, R. Valdagni, A. N. W. G. on Prostate Radiotherapy, et al. The impact of contouring uncertainty on rectal $3 \mathrm{D}$ dose-volume data: Results of a dummy run in a multicenter trial (airopros01-02). International Journal of Radiation Oncology* Biology* Physics, 57(2):573-579, 2003.

[8] A. Gegenfurtner, A. Siewiorek, E. Lehtinen, and R. Saljo. Assessing the quality of expertise differences 


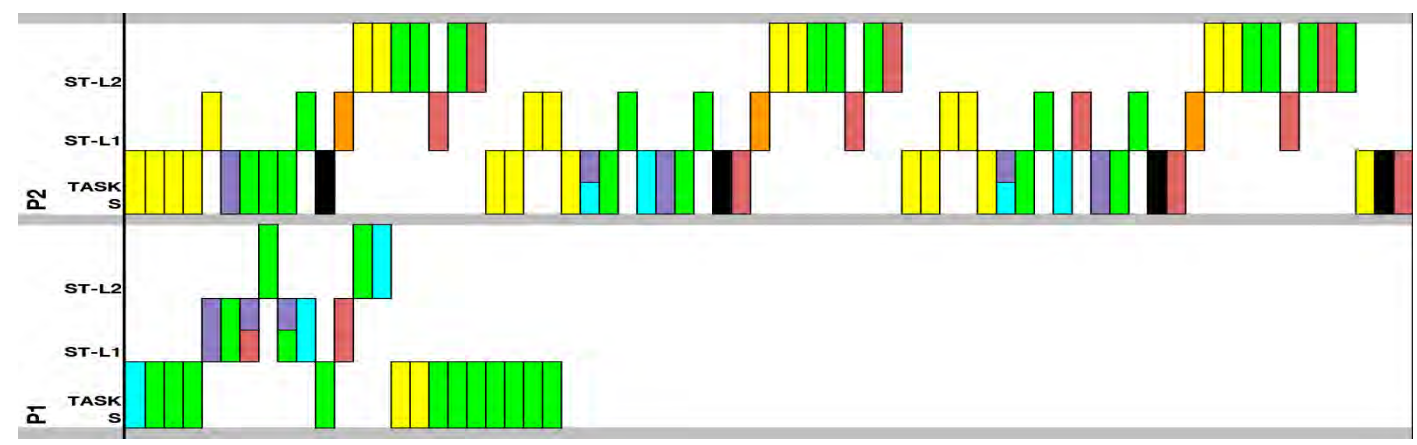

Figure 5: Task flow patterns for Participants P1 and P2.

in the comprehension of medical visualizations. Vocations and Learning, 6(1):37-54, 2013.

[9] Z. Guan, S. Lee, E. Cuddihy, and J. Ramey. The validity of the stimulated retrospective think-aloud method as measured by eye tracking, 2006.

[10] L. Hoyte, W. Ye, L. Brubaker, J. R. Fielding, M. E. Lockhart, M. E. Heilbrun, M. B. Brown, and S. K. Warfield. Segmentations of MRI images of the female pelvic floor: A study of inter-and intra-reader reliability. Journal of Magnetic Resonance Imaging, 33(3):684-691, 2011.

[11] A. Hyrskykari, S. Ovaska, P. Majaranta, K.-J. Räihä, and M. Lehtinen. Gaze path stimulation in retrospective think-aloud. Journal of Eye Movement Research, 2(4):1-18, 2008.

[12] C. Johnson, R. Moorhead, T. Munzner, H. Pfister, P. Rheingans, and T. S. Yoo. NIH/NSF visualization research challenges report. In Los Alamitos, Ca: IEEE Computing Society. Citeseer, 2006.

[13] T. Ju, Q.-Y. Zhou, and S.-M. Hu. Editing the topology of $3 \mathrm{D}$ models by sketching. In $A C M$ SIGGRAPH 2007 Papers, SIGGRAPH '07, New York, NY, USA, 2007. ACM.

[14] G. A. Klein, R. Calderwood, and D. Macgregor. Critical decision method for eliciting knowledge. Systems, Man and Cybernetics, IEEE Transactions on, 19(3):462-472, 1989.

[15] E. A. Krupinski. Current perspectives in medical image perception. Attention, Perception, $\mathcal{E S}^{\circ}$ Psychophysics, 72(5):1205-1217, 2010.

[16] U. Kuckartz. Maxqda: Qualitative data analysis. Berlin: VERBI software, 2007.

[17] R. Li, J. Pelz, P. Shi, C. O. Alm, and A. R. Haake. Learning eye movement patterns for characterization of perceptual expertise. In Proceedings of the Symposium on Eye Tracking Research and Applications, pages 393-396, New York, NY, USA, 2012. ACM, ETRA '12.

[18] M. R. Mahfouz, W. A. Hoff, R. D. Komistek, and D. A. Dennis. Effect of segmentation errors on $3 \mathrm{D}$-to-2D registration of implant models in x-ray images. Journal of biomechanics, 38(2):229-239, 2005.

[19] U. Neisser. Cognition and reality: Principles and implications of cognitive psychology. WH Freeman/Times Books/Henry Holt \& Co, 1976.

[20] S. D. Olabarriaga and A. W. Smeulders. Interaction in the segmentation of medical images: a survey. Medical Image Analysis, 5(2):127-142, 2001.

[21] A. Ramkumar, J. Dolz, H. A. Kirisli, S. Adebahr, T. Schimek-Jasch, U. Nestle, L. Massoptier, E. Varga, P. J. Stappers, W. J. Niessen, et al. User interaction in semi-automatic segmentation of organs at risk: a case study in radiotherapy. Journal of digital imaging, pages 1-14, 2015.

[22] A. Sanandaji, C. Grimm, R. West, and M. Parola. Where do experts look while doing 3D image segmentation. In Proceedings of the Ninth Biennial ACM Symposium on Eye Tracking Research 8 Applications, pages 171-174. ACM, 2016.

[23] L.-K. Soh and C. Tsatsoulis. Learning methodologies and discriminating visual cues for unsupervised image segmentation. In Seventeenth International Conference on Machine Learning: Workshop on Machine Learning of Spatial Knowledge, Palo Alto, CA, 2000.

[24] J. Van de Steene, N. Linthout, J. de Mey, V. Vinh-Hung, C. Claassens, M. Noppen, A. Bel, and G. Storme. Definition of gross tumor volume in lung cancer: inter-observer variability. Radiotherapy and Oncology, 62(1):37-49, 2002.

[25] S. K. Warfield, K. H. Zou, and W. M. Wells. Simultaneous truth and performance level estimation (staple): an algorithm for the validation of image segmentation. Medical Imaging, IEEE Transactions on, 23(7):903-921, 2004.

[26] O. Wirjadi. Survey of $3 D$ image segmentation methods, volume 35. ITWM, 2007. 